\title{
Evaluation of kinematic changes of intervertebral discs and the space available for the cord
}

\author{
Federico Canavese $^{1}$, Yann Philippe Charles ${ }^{2}$, Alain Dimeglio ${ }^{3}$ \\ ${ }^{1}$ Department of Pediatric Orthopedic Surgery, University Hospital Estaing, Clermont Ferrand, France; ${ }^{2}$ Department of Spine Surgery, University \\ Hospital of Strasbourg, Strasbourg, France; ${ }^{3}$ Faculty of Medicine, University of Montpellier, Montpellier, France \\ Correspondence to: Prof. Federico Canavese, MD, PhD. Department of Pediatric Orthopedic Surgery, University Hospital Estaing, 1 place Lucie et \\ Raymond Aubrac, Clermont Ferrand 63003, France. Email: canavese_federico@yahoo.fr. \\ Provenance: This is an invited Editorial commissioned by Section Editor Yan Yu (Department of Orthopaedics, Spine Surgery, Tongji Hospital, Tongji \\ University, Shanghai, China). \\ Comment on: Paholpak P, Shah I, Acevedo-Moreno LA, et al. Kinematic analysis of the space available for cord and disc bulging of the thoracic spine \\ using kinematic magnetic resonance imaging (kMRI). Spine J 2018;18:1122-7.
}

Submitted Aug 31, 2018. Accepted for publication Sep 10, 2018.

doi: 10.21037/atm.2018.09.15

View this article at: http://dx.doi.org/10.21037/atm.2018.09.15

Data on healthy human spinal cord morphometrics have been published for more than a century, using different measurement techniques and different anatomical landmarks. In recent years, there has been significant interest in the kinematic changes in disc bulging and the space available for the cord (SAC) in the cervical spine and the cauda equina in the lumbar spine (1-4). However, the kinematic changes in intervertebral discs and the SAC within the thoracic region have not been well analyzed and, as a result, the amount of movement of the thoracic spinal cord within the dural sac is still unknown. Thoracic disc herniation can cause myelopathy. Compared to cervical or lumbar disc herniation, it is an uncommon condition, accounting for $1 \%$ to $2.9 \%$ of all symptomatic herniated discs. In cases of myelopathy with weakness in the legs, and bladder and bowel disfunction, thoracic disc herniation might be indicated for surgical treatment (1).

The thoracic spine is considered to be a semi-rigid region of the human spine. Nevertheless, several studies have reported dynamic changes to the thoracic spine in terms of motion and the cross-sectional area of the thoracic spinal cord. The anatomical disposition of the thoracic vertebrae, ligaments, and their relationship to the rib cage play a central role in regulating the stability of the thoracic spine in flexion-extension, lateral bending, and axial rotation. Thoracic vertebrae are characterized by the coronal orientation of facet joints, which limits range of motion $(\mathrm{ROM})$ in flexion-extension. The intervertebral disc height is relatively narrow and the anterior longitudinal ligament represents a strong tension band in the sagittal concavity of the thoracic spine. These anatomical structures restrict the possibility for extension. Alternatively, the interspinous and supraspinous ligaments limit ROM in flexion and prevent anterior instability and kyphotic deformity $(5,6)$. Moreover, the articulation of the rib head with the vertebral bodies provides stability to the thoracic spine in lateral bending and axial rotation. Takeuchi et al., in particular, proved the biomechanical role of the intervertebral disc and the costovertebral joint in the stability of the thoracic spine, finding that the unilateral resection of the rib head joint after partial discectomy on the same side results in a significant decrease in thoracic spinal stability (7).

Although the thoracic spine is the most stable section of the spinal column, the thoracic spinal canal exhibits the narrowest SAC. The anatomical characteristics of the thoracic spine cause the spinal cord to become susceptible to compression when an angular kyphotic deformity is present, which can progress to symptomatic thoracic myelopathy. Furthermore, the thoracic segment of the spinal cord has the poorest blood supply as compared to the cervical spinal cord and the conus terminalis at the thoracolumbar junction. This makes the thoracic cord particularly vulnerable to ischemia, leading to anterior cord syndrome with pyramidal tract impairment, or even central cord syndrome. The clinician might consider that the prognosis for recovery of a thoracic neurologic deficit 
remains poor, especially for complete syndromes (ASIA A), since the blood supply to the thoracic spinal cord is deficient $(8,9)$.

The dynamic nature of the spinal column and its mobility across multiple segments is difficult to depict with a static imaging modality. Conventional magnetic resonance imaging (MRI) of the spine is usually performed in a supine position, which can result in false negative findings. The advent of kinematic MRI (kMRI) has allowed investigators to image patients in a weight-bearing position and to analyze structural displacements and distortions of the anatomy of intervertebral discs, neural elements, ligaments, and facets by varying their positions, thus revealing abnormalities that are potentially missed by traditional MRI studies $(10,11)$.

The significant work by Paholpak et al., published in the July 2018 issue of The Spine Fournal, is a remarkable attempt to evaluate the kinematic changes of intervertebral discs and the SAC within the thoracic spine using kMRI (12). This study aimed to quantify the variations of disc bulging and the SAC for three load scenarios: seated upright neutral position $\left(0^{\circ}\right)$, flexion $\left(40^{\circ}\right)$, and extension $\left(-20^{\circ}\right)$. The authors of this work reported that the thoracic spinal canal tended to be narrowest in flexion, with reduced SAC as compared to the neutral position, and widest in extension, with a concomitant increase of the SAC. Not surprisingly, disc bulging showed a trend that was opposite of the SAC, with the greatest mean disc bulging occurring in flexion and the smallest bulging occurring in extension. These findings were particularly evident at mid- and lower thoracic spine levels, particularly at T8-T9 and T9-T10, which are located around the sagittal kyphotic apex, and T11-T12, which correspond to the transitional anatomical area between the true ribs and the false floating ribs. In a previous study, Morita et al. showed that anteroposterior diameter and the cross-sectional area of the dural sac were smaller in flexion than in extension at the upper and middle thoracic spine, reporting that the segmental kyphotic angle, rather than the segmental range of movement, is the most relevant factor affecting dimensions of the dural sac and spinal cord (6). Paholpak et al. hypothesized that kyphotic deformities could be one of the possible etiologies for neurogenic deterioration in patients with symptomatic thoracic myelopathy, since kyphosis can be assimilated in thoracic spine flexion (12). This may also explain why thoracic myelopathy might be exacerbated in flexion and be improved in neutral and extension positions. The role of segmental kyphotic deformity has a major clinical significance. This includes congenital kyphosis, neurofibromatosis, and other syndromes leading to angular kyphotic deformities. Posttraumatic kyphosis, as well as vertebral body collapse in osteoporosis, myeloma, or vertebral metastases represent other etiologies that can lead to segmental kyphosis and anterior cord syndrome or complete paralysis in severe cases (13).

The findings provided by Paholpak et al. are compelling and the paper potentially opens the door for more research on this topic. However, some points deserve additional research and discussion. This is a cross-sectional study of a limited number of patients that could not take into account the large intra- (up to $30 \%$ ) and inter-individual variability (up to $13 \%$ ) of both morphology and dimensions of the spinal cord. Moreover, significant differences related to age and postmortem state have also been identified $(1,5,6,12)$. In particular, age appears to be a potential confounding factor, as spine segmental motion and flexibility tend to decrease with age. With aging, the bone quality worsens and impacts both the sagittal alignment and the motion of the spinal column, adding an additional potential bias to the results. The thoracic spinal cord changes in shape and position in the dural sac during positional changes. In flexion, the thoracic cord tends to move anteriorly closer to the posterior vertebral body and the intervertebral disc. How much of this anterior displacement is tolerated? Is it the same at all thoracic spinal levels? Also, with aging, the intervertebral discs undergo biochemical modifications of the nuclear matrix as the balance between synthesis and degradation of the matrix is disturbed. Furthermore, genetic predispositions, as well as vascular factors, have been advocated in the process of disc degeneration (14). MRI studies comparing the aspect of the disc in a supine position and under axial load compression, as in standing position, have demonstrated that load bearing would induce a circumferential bulging of the disc $(3,11,13)$. Disc bulging and herniation, ossified posterior longitudinal ligament, calcified ligamentum flavum, and diffuse idiopathic skeletal hyperostosis with major bone formation at thoracic spinal levels represent factors that might decrease the SAC and potentially lead to symptomatic thoracic myelopathy. The nerve root may also be compressed at the level of the lateral recess or at the intervertebral foramen. Nonetheless, positional changes in the sagittal plane might play a minor role in the thoracic spine as compared to the cervical or the lumbar spine. Bulging of the intervertebral disc and the ligamentum flavum narrow the spinal canal in extension, which mainly has clinical relevance in cervical and lumbar 
dynamic stenosis.

Growth might be considered further in children and adolescents, since anatomical relationships and dimensions change over time. Dimeglio et al. have shown that the spinal canal reaches its mature size following the closure of the posterior and neurocentral synchondroses; by the age of five the spinal canal has reached approximately $95 \%$ of its definitive size. This growth pattern does not match the growth of the vertebral body, which shows a constant growth rate from birth to skeletal maturity (9). Once skeletal maturity is reached, age has a limited influence on canal size variations and on the $\operatorname{SAC}(5,9,13,15)$. Moreover, most of the previous works, including those discussed here, have treated the spinal canal as a circle and have focused on anterior-posterior distance only. As a result, thoracic spine dimensions during the different stages of life (birth, childhood, adulthood, and old age) are not fully known. In practice, the relationship between spinal canal dimensions, vertebral body heights, disc, ligament, and cord elasticity are clinically relevant in children. Spinal cord injury without radiographic abnormality (SCIWORA) represents a severe posttraumatic condition that is related to specific anatomical relationships between the cord and the spine during growth. In these young injured patients, the cord elasticity is lower than the elasticity of cartilaginous and disco-ligamentous elasticity of spinal structures, which might induce cord ischemia and subsequent paralysis.

Improving knowledge concerning the average size and variability of the human spinal cord and SAC can be of importance when treating pathological conditions in the spinal cord. Further work is needed to reinforce the findings of Paholpak et al. $(1,12)$. In future analyses, it is important to include not only the anatomical factors, such as the amount of bulging and SAC, but the location of the bulging; lateral protrusions tend to produce radiculopathies while central disc protrusions are susceptible to producing symptomatic myelopathy. Furthermore, kMRI changes might improve our understanding of thoracic herniated discs.

When considering optimal care for patients with thoracic disc herniation and myelopathy, clinical evidence is relatively sparse. It makes sense to consider surgical decompression by an anterior approach or by a posterior costotransversectomy in patients with progressive neurologic impairment. However, the poor blood supply of the cord and the risk for postoperative neurologic deterioration need to be considered. The kinematic diagnostic approach to thoracic herniated discs is not routinely considered today. Usually the surgeon looks for calcifications of the herniated disc on CT, which represents an indirect sign for an older fixed herniation that might not change shape in flexion-extension. The kMRI might represent an additional diagnostic tool, which would enable the researcher to determine if positional changes decrease the SAC, which might worsen the extent of spinal cord compression and neurologic impairment. To date, it remains unclear if this type of kinematic imaging could improve our understanding of soft disc herniation, thoracic stenosis, and myelopathy. It might be interesting to further study the role of the $\mathrm{kMRI}$ in combination with clinical neurological testing, motor and somatosensory evoked potentials, and spinal cord tractography. New findings could influence our future treatment algorithms for thoracic disc herniation and stenosis.

\section{Acknowledgements}

None.

\section{Footnote}

Conflicts of Interest: The authors have no conflicts of interest to declare.

\section{References}

1. Paholpak P, Nazareth A, Barkoh K, et al. Space Available for Cord, Motion, and disc degeneration at the adjacent segments level of degenerative cervical spondylolisthesis using kinematic MRI. J Clin Neurosci 2017;45:89-99.

2. Parent EC, Videman T, Battié MC. The effect of lumbar flexion and extension on disc contour abnormality measured quantitatively on magnetic resonance imaging. Spine 2006;31:2836-42.

3. Schmid MR, Stucki G, Duewell S, et al. Changes in cross-sectional measurements of the spinal canal and intervertebral foramina as a function of body position: In vivo studies on an open-configuration MR system. AJR Am J Roentgenol 1999;172:1095-102.

4. Pfirmann CW, Metzdorf A, Zanetti M, et al. Magnetic resonance classification of lumbar intervertebral disc degeneration. Spine (Phila Pa 1976) 2001;26:1873-8.

5. Fradet L, Arnoux PJ, Ranjeva JP, et al. Morphometrics of the entire human spinal cord and spinal canal measured from in vivo high-resolution anatomical magnetic resonance imaging. Spine (Phila Pa 1976) 2014;39:E262-9.

6. Morita D, Yukawa Y, Nakashima H, et al. Dynamic 
changes in the cross-sectional area of the dural sac and spinal cord in the thoracic spine. Eur Spine J 2017;26:64-70.

7. Takeuchi T, Abumi K, Shono Y, et al. Biomechanical role of the intervertebral disc and costovertebral joint in stability of the thoracic spine. A canine model study. Spine (Phila Pa 1976) 1999;24:1414-20.

8. Charles YP, Barbe B, Beaujeux R, et al. Relevance of the anatomical location of the Adamkiewicz artery in spine surgery. Surg Radiol Anat 2011;33:3-9.

9. Dimeglio A, Canavese F. The growing spine: how spinal deformities influence normal spine and thoracic cage growth. Eur Spine J 2012;21:64-70.

10. Michelini G, Corridore A, Torlone S, et al. Dynamic MRI in the evaluation of the spine: state of the art. Acta Biomed 2018;89:89-101.

11. Hansson T, Suzuki N, Hebelka H, et al. The narrowing

Cite this article as: Canavese F, Charles YP, Dimeglio A. Evaluation of kinematic changes of intervertebral discs and the space available for the cord. Ann Transl Med 2018;6(Suppl 1):S22. doi: 10.21037/atm.2018.09.15 of the lumbar spinal canal during loaded MRI: the effects of the disc and ligamentum flavum. Eur Spine J 2009;18:679-86.

12. Paholpak P, Shah I, Acevedo-Moreno LA, et al. Kinematic analysis of the space available for cord and disc bulging of the thoracic spine using kinematic magnetic resonance imaging (kMRI). Spine J 2018;18:1122-7.

13. Frostell A, Hakim R, Thelin EP, et al. A Review of the Segmental Diameter of the Healthy Human Spinal Cord. Front Neurol 2016;7:238.

14. Hadjipavlou AG, Tzermiadianos MN, Bogduk N, et al. The pathophysiology of disc degeneration: a critical review. J Bone Joint Surg Br 2008;90:1261-70.

15. Ulbrich EJ, Schraner C, Boesch C, et al. Normative MR cervical spinal canal dimensions. Radiology 2014;271:172-82. 